\title{
An equivalence theorem for a differential information economy
}

\author{
Carlos Hervés-Beloso ${ }^{\mathrm{a}}$, Emma Moreno-García ${ }^{\mathrm{b}}$, \\ Nicholas C. Yannelis ${ }^{\mathrm{c}, *}$ \\ a RGEA, Facultad de Económicas, Universidad de Vigo, Spain \\ ${ }^{\mathrm{b}}$ Facultad de Economía y Empresa, Universidad de Salamanca, Spain \\ ${ }^{c}$ Department of Economics, University of Illinois, 1206 S. 6th Street, Champaign, IL 61820, USA
}

Received 24 September 2003; received in revised form 26 November 2003; accepted 20 January 2004

Available online 16 February 2005

\begin{abstract}
In a pure exchange economy with differential information and a finite set of traders, physical commodities and states of nature, we characterize the Walrasian expectations or Radner equilibria by using the veto power of the grand coalition. We prove that an allocation $x$ is a Radner equilibrium allocation if and only if it is "privately non-dominated" by the grand coalition in every economy obtained by perturbing the original initial endowments in the direction of $x$. The first and second welfare theorems become particular cases of our main result. Since the deterministic Arrow-DebreuMcKenzie model is a special case of the differential information economy model we also provide a new characterization of the Walrasian equilibria.
\end{abstract}

(C) 2004 Elsevier B.V. All rights reserved.

JEL classification: D51; D82; D11

Keywords: Differential information economy; Radner equilibrium; Privately non-dominated allocations; Private core

* Corresponding author. Tel.: +1 217333 0120; fax: +1 2172446678.

E-mail addresses: cherves@uvigo.es (C. Hervés-Beloso), emmam@usal.es (E. Moreno-García), nyanneli@uiuc.edu (N.C. Yannelis). 


\section{Introduction}

The classical Arrow-Debreu-McKenzie (A-D-M) model consists of a finite number of commodities and a finite number of agents, each of whom is characterized by her preferences and initial endowments. Arrow and Debreu introduced uncertainty into the classical A-D-M model and showed (see Debreu, 1959, Chapter 7) how the deterministic results are still valid in the presence of uncertainty. Radner (1968) introduced differential (asymmetric) information into the A-D-M model. In particular, an exchange economy with differential information (or a Radner-type economy) consists of a finite set of agents, each of whom is characterized by a random utility function, a random initial endowment, a private information set, and a prior. For such an economy Radner (1968) defined a notion of a Walrasian expectations equilibrium, here called Radner equilibrium. This notion is analogous to the Walrasian equilibrium in the Arrow-Debreu-McKenzie deterministic model. The Radner equilibrium notion is of interest because it captures trades under asymmetric information. In such an economy, agents maximize ex-ante expected utility subject to their budget constraint. However, each agent's allocation is measurable with respect to her own private information, and thus, all choices made reflect the informational asymmetries. It should be noted that the Radner equilibrium differs from the rational expectations equilibrium (REE) (e.g., Radner, 1979), which is an interim concept allowing prices to reveal some or all of the private information in the economy. A major criticism of the REE is that it does not provide an adequate explanation as to how prices reveal the same information to agents who are differentially informed and, therefore, prices do not reflect the differential information of agents. This is not an issue for the Radner equilibrium since decisions are made in an ex-ante stage; however, since net trades are private information measurable for each agent, the equilibrium outcome reflects the asymmetric information. Furthermore, the Radner equilibrium exists under the standard assumptions which guarantee the existence of the deterministic Walrasian equilibrium, whereas the REE may not exist in well-behaved economies (e.g., Kreps, 1977). In addition, in the absence of free disposal, the Radner equilibrium is coalitional Bayesian incentive compatible (that is, no coalition can misreport the realized state of nature to the complementary set of agents and become better off) and can be supported as a perfect Bayesian equilibrium (see Glycopantis et al., 2003), contrary to the REE. Therefore, the Radner equilibrium seems to be an appealing concept.

The purpose of this paper is to study further the Radner equilibrium concept and obtain some new results.

The main result in this paper provides a characterization of Radner equilibria (and, in particular, of Walrasian equilibria) in terms of non-dominated allocations. The notion of non-dominated allocation that we consider states that it is not possible for the grand coalition to redistribute their initial endowments using their own private information and make each member of the grand coalition better off (in terms of their ex-ante expected utility). Since agents do not necessarily share their own private information, we call those allocations privately non-dominated allocations. Thus, privately non-dominated allocations have similar features to the (ex-ante) private core (Yannelis, 1991). In particular, the private core is contained in the set of all privately non-dominated allocations and, therefore, the existence of private core allocations implies the existence of private non-dominated allocations. 
It should be remarked that the characterization of Radner equilibria as privately nondominated allocations allows us to obtain as immediate consequences the first and second welfare theorems for differential information economies.

A differential information economy with a finite number of agents can be interpreted as a continuum differential information economy in which only a finite number of different agents' characteristics can be distinguished. We show that a price-consumption pair is a Radner equilibrium for a discrete economy with differential information if and only if it is also a Radner equilibrium for the associated continuum differential information economy. Thus, these continuum of agents differential information economies and discrete ones can be considered equivalent with respect to Radner equilibria.

Our characterization of Radner equilibrium is new and relies on an extension of a result of Vind (1972) to a differential information economy setting. The argument is not straightforward and requires the private core-equivalence theorem (Einy et al., 2001). However, our proof has a big payoff. Indeed, not only do we obtain as corollaries the welfare theorems for differential information economies, but we also obtain new insights for the deterministic Walrasian model. In particular, since the deterministic Arrow-Debreu-McKenzie model is a special case of the differential information economy model, one derives a new characterization of the Walrasian equilibria which yields the first and second welfare theorem for symmetric information economies as simple corollaries and also provides a new proof for the second welfare theorem. Actually, we characterize Walrasian equilibria by exploiting the veto power of the grand coalition. Related results characterizing Walrasian equilibria by using the veto power are those by Debreu and Scarf (1963) and Aubin (1979). Indeed, Debreu and Scarf (1963) show the coincidence between the set of Walrasian allocations and the set of Edgeworth equilibria and Aubin (1979) proves that the fuzzy core characterizes Walrasian equilibrium. These well-known equivalence results differ substantially from the one provided in this paper. The Debreu-Scarf and Aubin arguments enlarge the set of blocking coalitions in order to obtain the equilibrium allocations; the former enlarge coalitions replicating the economy and the latter allowing the participation of the agents with any rate of their endowments. However, we provide a characterization of equilibria by considering the veto power of just one coalition, namely the grand coalition, whereas enlarging the possible redistribution of endowments (by perturbing slightly the original initial endowments following a precise direction).

The paper proceeds as follows. Section 2 contains the main concepts in a differential information economy with finitely many agents. Section 3 focuses on the interpretation of finite differential information economies as continuum of agents differential information economies with a finite number of types of agents. Moreover, in this section, an extension of Vind's (1972) result is given for a differential information economy. Section 4 contains a characterization of Radner equilibrium allocations as privately non-dominated allocations. Finally, some concluding remarks are summarized in Section 5. Technical proofs are included in the Appendix A.

\section{Differential information economies with a finite number of agents}

Let us consider a Radner-type exchange economy $\mathcal{E}$ with differential information (see Radner, 1968, 1982). Let $(\Omega, \mathcal{F})$ be a measurable space, where $\Omega$ denotes the states of 
nature and the algebra $\mathcal{F}$ denotes the set of all events. Hence, $(\Omega, \mathcal{F})$ describes the exogenous uncertainty. The set of states of nature, $\Omega$, is finite and there is a finite number of goods, $\ell$, in each state. $N$ is the set of $n$ traders or agents and $\mathbb{R}_{+}^{\ell}$ will denote the commodity space which is the positive orthant of $\mathbb{R}^{\ell}$.

The economy extends over two time periods $\tau=0,1$. Consumption takes place at $\tau=1$. At $\tau=0$, there is uncertainty over the states of nature and agents make contracts (agreements) that may be contingent on the realized state of nature at period $\tau=1$ (that is, ex-ante contract arrangement).

A differential information exchange economy $\mathcal{E}$ with a finite number of agents is defined by $\left\{\left((\Omega, \mathcal{F}), X_{i}, \mathcal{F}_{i}, U_{i}, e_{i}, q\right): i=1, \ldots, n\right\}$, where:

1. $X_{i}: \Omega \rightarrow 2^{\mathbb{R}_{+}^{\ell}}$ is the set valued function denoting the random consumption set of agent $i$

2. $\mathcal{F}_{i}$ is a partition of $\Omega$, denoting the private information of agent $i$;

3. $U_{i}: \Omega \times \mathbb{R}_{+}^{\ell} \rightarrow \mathbb{R}$ is the random utility function of agent $i$;

4. $e_{i}: \Omega \rightarrow \mathbb{R}_{+}^{\ell}$ is the random initial endowment of agent $i$, assumed to be constant on elements of $\mathcal{F}_{i}$, with $e_{i}(\omega) \in X_{i}(\omega)$ for all $\omega \in \Omega$;

5. $q$ is a probability function on $\Omega$ giving the (common) prior of every agent. It is assumed that $q$ is positive on all elements of $\Omega$.

We will refer to a function with domain $\Omega$, constant on elements of $\mathcal{F}_{i}$, as $\mathcal{F}_{i}$-measurable, although, strictly speaking, measurability is with respect to the $\sigma$-algebra generated by the partition. We can think of such a function as delivering information to trader $i$, who can not discriminate between the states of nature belonging to any element of $\mathcal{F}_{i}$.

For any $x: \Omega \rightarrow \mathbb{R}_{+}^{\ell}$, the ex-ante expected utility of agent $i$ is given by

$$
V_{i}(x)=\sum_{\omega \in \Omega} U_{i}(\omega, x(\omega)) q(\omega)
$$

Let $L_{X_{i}}$ denote the set of all $\mathcal{F}_{i}$-measurable selections from the random consumption set of agent $i$, that is:

$$
L_{X_{i}}=\left\{x_{i}: \Omega \rightarrow \mathbb{R}^{\ell} \text {, such that } x_{i} \text { is } \mathcal{F}_{i} \text {-measurable and } x_{i}(\omega) \in X_{i}(\omega) \text { for all } \omega\right\}
$$

Let $L_{X}=\prod_{i=1}^{n} L_{X_{i}}$. Any element $x$ in $L_{X}$ is called an allocation. An allocation $x \in L_{X}$ is said to be feasible if $\sum_{i=1}^{n} x_{i} \leq \sum_{i=1}^{n} e_{i}$.

A coalition $S \subset N$ privately blocks an allocation $x \in L_{X}$ if there exists $\left(y_{i}\right)_{i \in S} \in \prod_{i \in S} L_{x_{i}}$ such that $\sum_{i \in S} y_{i} \leq \sum_{i \in S} e_{i}$ and $V_{i}\left(y_{i}\right)>V_{i}\left(x_{i}\right)$ for every $i \in S$.

The private core of the differential information exchange economy $\mathcal{E}$ is the set of all feasible allocations which are not privately blocked by any coalition.

Next, we shall define a Walrasian equilibrium notion in the sense of Radner. In order to do so, we need the following definitions. A price system is an $\mathcal{F}$-measurable, non-zero 
function $p: \Omega \rightarrow \mathbb{R}_{+}^{\ell}$. For a price system $p$, the budget set of agent $i$ is given by

$$
B_{i}(p)=\left\{x_{i} \in L_{X_{i}}, \text { such that } \sum_{\omega \in \Omega} p(\omega) x_{i}(\omega) \leq \sum_{\omega \in \Omega} p(\omega) e_{i}(\omega)\right\}
$$

Notice that traders must balance the budget ex-ante.

Definition 2.1. A pair $(p, x)$, where $p$ is a price system and $x=\left(x_{1}, \ldots, x_{n}\right) \in L_{X}$ is an allocation, is a Radner equilibrium if

(i) for all $i$, the consumption function maximizes $V_{i}$ on $B_{i}$,

(ii) $\sum_{i=1}^{n} x_{i} \leq \sum_{i=1}^{n} e_{i}$ (free disposal), and

(iii) $\sum_{\omega \in \Omega} p(\omega) \sum_{i=1}^{n} x_{i}(\omega)=\sum_{\omega \in \Omega} p(\omega) \sum_{i=1}^{n} e_{i}(\omega)$.

Radner equilibrium is an ex-ante concept. Notice that we assume free disposal. It is well known that if we impose the condition of non-free disposal then a Radner equilibrium might not exist with positive prices (see, for example, Glycopantis et al., 2003). However, allowing for negative prices one can dispense with the free disposal assumption.

Definition 2.2. An allocation $x \in L_{X}$ is privately dominated (or privately blocked by the grand coalition) in the economy $\mathcal{E}$ if there exists a feasible allocation $y \in L_{X}$, such that $V_{i}\left(y_{i}\right)>V_{i}\left(x_{i}\right)$ for every $i=1, \ldots, n$.

Observe that to be feasible and to be dominated are independent conditions for an allocation $x \in L_{X}$. According to the definition above, a (privately) Pareto optimal allocation is a feasible and non-dominated allocation.

Note also that despite the fact that the whole coalition of agents get together they do not share their own information. To the contrary, the redistribution of the initial endowments is based only on their own private information. Hence, a feasible and non-dominated allocation reflects the private information of each agent and has the property that the coalition of all the agents can not redistribute their initial endowments, based on their own private information, and make every individual better off.

In this paper, we will assume that $X_{i}(\omega)=\mathbb{R}_{+}^{\ell}$ for every agent $i$ and for every $\omega \in \Omega$; and we state the following assumptions on endowments and preferences:

(A.1). $e_{i}(\omega) \gg 0$ for all $i$ and for all $\omega \in \Omega$.

(A.2). For all $i$ and $\omega, U_{i}(\omega, \cdot): \mathbb{R}_{+}^{\ell} \rightarrow \mathbb{R}$ is continuous, strictly monotone and concave.

(A.3). For all $i, U_{i}(\cdot, x): \Omega \rightarrow \mathbb{R}$ is measurable.

Remark. Assumption (A.1) is often replaced by $\sum_{i=1}^{n} e_{i}(\omega) \gg 0$ for all $\omega \in \Omega$ together with irreducibility (i.e., the endowment of every coalition is desired). 


\section{A continuum approach}

\subsection{Radner equilibria in continuum economies}

In this section, we interpret differential information economies with $n$ agents as continuum economies where the $i$ th agent is the representative of infinitely many identical agents (see García-Cutrín and Hervés-Beloso, 1993 for the case of Arrow-Debreu-McKenzie economies). For it, let us associate to the differential information economy $\mathcal{E}$ (described in the previous section) a continuum economy $\mathcal{E}_{\mathrm{c}}$ with differential information in which only a finite number of different agents can be distinguished.

Given $\mathcal{E} \equiv\left\{\left((\Omega, \mathcal{F}), X_{i}, \mathcal{F}_{i}, U_{i}, e_{i}, q\right): i=1, \ldots, n\right\}$, the associated atomless economy $\mathcal{E}_{\mathrm{c}}$ is defined as follows. The set of agents is represented by the unit real interval $I=[0,1]=\bigcup_{i=1}^{n} I_{i}$, where $I_{i}=\left[\frac{i-1}{n}, \frac{i}{n}\right)$, if $i \neq n$, and $I_{n}=\left[\frac{n-1}{n}, 1\right]$. We consider the Lebesgue measure $\mu$ on the Borel subsets of $I$. Each agent $t \in I$ is characterized by her private information which is described by a partition $\mathcal{F}_{t}$ of $\Omega$, where $\mathcal{F}_{t}=\mathcal{F}_{i}$ for every $t \in I_{i}$; her consumption set $X_{t}(\omega)=\mathbb{R}_{+}^{\ell}$ for every $\omega \in \Omega$; her random initial endowment $e(t, \cdot)=e_{i}$ for every $t \in I_{i}$ and her random utility function $U_{t}=U_{i}$. Hence, the ex-ante expected utility function for every agent $t \in I_{i}$ is $V_{t}=V_{i}$. We will refer to agents belonging to the subinterval $I_{i}$ as agents of type $i$.

An allocation is a function $f: I \times \Omega \rightarrow \mathbb{R}_{+}^{\ell}$, such that for every $\omega \in \Omega$, the function $f(\cdot, \omega)$ is $\mu$ integrable on $I$ and for almost all $t \in I$ the function $f(t, \cdot)$ is $\mathcal{F}_{t}$-measurable.

An allocation $f$ is feasible for the economy $\mathcal{E}_{\mathrm{c}}$ if $\int_{I} f(t, \omega) \mathrm{d} \mu(t) \leq \int_{I} e(t, \omega) \mathrm{d} \mu(t)$ for all $\omega \in \Omega$.

A coalition in $\mathcal{E}_{\mathrm{c}}$ is a Borel subset of $I$. A coalition $S \subset I$, with $\mu(S)>0$, privately blocks an allocation $f$ if there exists $g: S \times \Omega \rightarrow \mathbb{R}_{+}^{\ell}$ such that $g(t, \cdot)$ is $\mathcal{F}_{t}$-measurable for all $t \in S, \int_{S} g(t, \omega) \mathrm{d} \mu(t) \leq \int_{S} e(t, \omega) \mathrm{d} \mu(t)$ for every $\omega \in \Omega$ and $V_{t}(g(t, \cdot))>V_{t}(f(t, \cdot))$ for every $t \in S$.

The private core of the economy $\mathcal{E}_{\mathrm{c}}$ is the set of feasible allocations which are not privately blocked by any coalition.

Note that given a price system $p: \Omega \rightarrow \mathbb{R}_{+}^{\ell}$, the budget set of agent $t \in I$ is $B_{t}(p)=$ $B_{i}(p)$ for every $t \in I_{i}$.

Definition 3.1. A competitive equilibrium (or Radner equilibrium) for the continuum economy $\mathcal{E}_{\mathrm{c}}$ is a pair $(p, f)$, where $p$ is a price system and $f$ is a feasible allocation such that:

(i) for almost all $t \in I$ the function $f(t, \cdot)$ maximizes $V_{t}$ on $B_{t}(p)$, and

(ii) $\sum_{\omega \in \Omega} p(\omega) \int_{I} f(t, \omega) \mathrm{d} \mu(t)=\sum_{\omega \in \Omega} p(\omega) \int_{I} e(t, \omega) \mathrm{d} \mu(t)$.

Remark. It follows from the continuity of $U_{i}(\omega, x)$ in $x$ and measurability in $\omega$ that $U_{i}(\cdot, \cdot)$ is jointly measurable. Hence, under (A.2) and (A.3), the associated continuum economy $\mathcal{E}_{\mathrm{c}}$ satisfies all the assumptions of the equivalence theorem of Radner equilibria and private core (see Einy et al., 2001).

Let us consider the differential information economy $\mathcal{E}$ with $n$ agents and the associated continuum economy $\mathcal{E}_{\mathrm{c}}$ with $n$ different types of agents. An allocation $x=\left(x_{1}, \ldots, x_{n}\right)$ in the economy $\mathcal{E}$ can be interpreted as an allocation $f$ in $\mathcal{E}_{\mathrm{c}}$, where $f$ is the step function defined 
by $f(t, \cdot)=x_{i},(i=1,2, \ldots, n)$ for every agent $t \in I_{i}$. Reciprocally, an allocation $f$ in $\mathcal{E}_{\mathrm{c}}$ can be interpreted as an allocation $x=\left(x_{1}, \ldots, x_{n}\right)$ in $\mathcal{E}$, where $x_{i}=\frac{1}{\mu\left(I_{i}\right)} \int_{I_{i}} f(t, \cdot) \mathrm{d} \mu(t)$.

We will show that the continuum and the discrete approach can be considered equivalent with respect to Radner equilibrium. In order to prove this result we will need the following Lemma.

Lemma 3.1. Let $S \subset I_{i},(i=1,2, \ldots, n)$ with $\mu(S)>0$, and $z: \Omega \rightarrow \mathbb{R}_{+}^{\ell}$. Let $f: S \times$ $\Omega \rightarrow \mathbb{R}_{+}^{\ell}$ be a Lebesgue integrable function such that $V_{i}(f(t, \cdot))>V_{i}(z)$ for every $t \in S$. Then, under Assumption (A.2), $V_{i}(h)>V_{i}(z)$, where $h(\omega)=\frac{1}{\mu(S)} \int_{S} f(t, \omega) \mathrm{d} \mu(t)$.

Proof. The proof follows from the concavity of the functions $V_{i}$ and Jensen's inequality. (See García-Cutrín and Hervés-Beloso, 1993, for a proof of this result, where a weaker convexity assumption on preferences is required).

Notice that the lemma is still true if in the hypothesis and in the statement we substitute $>$ for $\geq$.

We are now ready to state our first result.

Theorem 3.1. Assume that, for every $i=1, \ldots, n,(\mathrm{~A} .2)$ holds for the random utility function $U_{i}$. The following statements hold:

If $(x, p)$ is a Radner equilibrium for the discrete economy $\mathcal{E}$, then $(f, p)$ is a Radner equilibrium for the associated continuum economy $\mathcal{E}_{\mathrm{c}}$, where $f(t, \cdot)=x_{i}$ if $t \in I_{i}$.

Reciprocally, if $(f, p)$ is a Radner equilibrium for the associated continuum economy $\mathcal{E}_{\mathrm{c}}$, then $(x, p)$ is a Radner equilibrium for the economy $\mathcal{E}$, where $x_{i}=\frac{1}{\mu\left(I_{i}\right)} \int_{I_{i}} f(t, \cdot) \mathrm{d} \mu(t)$.

Proof. Let $\left(\left(x_{1}, \ldots, x_{n}\right), p\right)$ be a Radner equilibrium for $\mathcal{E}$. Then, for every state $\omega \in \Omega$, $\int_{I} f(t, \omega) \mathrm{d} \mu(t)=\sum_{i=1}^{n} \mu\left(I_{i}\right) x_{i}(\omega) \leq \sum_{i=1}^{n} \mu\left(I_{i}\right) e_{i}(\omega)=\int_{I} e(t, \omega) \mathrm{d} \mu(t)$ and, for all $t \in$ $I_{i}$, the consumption function $f(t, \cdot)$ maximizes $V_{t}$ on $B_{t}(p)=B_{i}(p)$. Therefore, $(f, p)$ is a Radner equilibrium for the continuum economy $\mathcal{E}_{\mathrm{c}}$.

Conversely, let $(f, p)$ be a Radner equilibrium for $\mathcal{E}_{\mathrm{c}}$. Then, $x=\left(x_{1}, \ldots, x_{n}\right)$, with $x_{i}=\frac{1}{\mu\left(I_{i}\right)} \int_{I_{i}} f(t, \cdot) \mathrm{d} \mu(t)$, is a feasible allocation in the economy $\mathcal{E}$. Since $\sum_{\omega \in \Omega} p(\omega) x_{i}(\omega)=\sum_{\omega \in \Omega} 1 / \mu\left(I_{i}\right) \int_{I_{i}} p(\omega) f(t, \omega) \mathrm{d} \mu(t) \leq \sum_{\omega \in \Omega} p(\omega) e_{i}(\omega)$, we can deduce that $x_{i} \in B_{i}(p)$ for every agent $i$. If $V_{i}(z)>V_{i}\left(x_{i}\right)$ then $V_{i}(z)>V_{i}(f(t, \cdot))$ for every $t \in S \subset I_{i}, \mu(S)>0$; and thus $\sum_{\omega \in \Omega} p(\omega) z(\omega)>\sum_{\omega \in \Omega} p(\omega) e_{i}(\omega)$. Otherwise, observe that if $V_{i}(z) \leq V_{i}(f(t, \cdot))$ for almost all $t \in I_{i}$, then by Lemma 3.1, $V_{i}(z) \leq V_{i}\left(x_{i}\right)$.

\subsection{An extension of Vind's theorem}

Three notes in the same issue of Econometrica gave a sharp and novel interpretation to Aummann's (1964) core-Walras equivalence result. Schmeidler (1972) showed that, in an atomless economy, with finitely many commodities, any allocation that is not blocked by "small" coalitions is in the core. Grodal (1972) showed that we can further restrict the set of coalitions to those consisting of finitely many arbitrarily small sets of agents with similar characteristics. Finally, Vind (1972) showed that it is enough to consider the blocking power of arbitrarily large coalitions in order to obtain the core.

The proof of our main result requires an extension of Vind's (1972) to differential information economies. To this end, we need the following Lemma. 
Lemma 3.2. Assume that assumption (A.1) holds and utility functions are continuous and strictly monotone. Then, if the allocation $f$ does not belong to the private core of the associated continuum economy $\mathcal{E}_{\mathrm{c}}$, it is privately blocked by a coalition A via an allocation $g$ with $\int_{A}(e(t, \omega)-g(t, \omega)) \mathrm{d} \mu(t) \gg 0$, for every $\omega \in \Omega$.

Proof. See Appendix A.

The next proposition extends the results of Schmeidler (1972) and Vind (1972) to cover the private core.

Proposition 3.1. Assume that (A.1)-(A.3) hold. If a feasible allocation $f$ is not in the private core of the associated continuum economy $\mathcal{E}_{\mathrm{c}}$ then for any $\alpha$, with $0<\alpha<1$ there exists a private blocking coalition $S$ with $\mu(S)=\alpha$.

Proof. Let $f$ be a feasible allocation which is privately blocked by a coalition $A \subset I$ via $g$. Then, $g(t, \cdot)$ is $\mathcal{F}_{t}$-measurable for all $t \in A, \int_{A} g(t, \omega) \mathrm{d} \mu(t) \leq \int_{A} e(t, \omega) \mathrm{d} \mu(t)$ for every $\omega \in \Omega$ and $V_{t}(g(t, \cdot))>V_{t}(f(t, \cdot))$ for every $t \in A$. In view of Lemma 3.2, we can consider $g: A \times \Omega \rightarrow \mathbb{R}_{+}^{\ell}$ such that, $\int_{A}(g(t, \omega)-e(t, \omega)) \mathrm{d} \mu(t)=-z(\omega)$, with $z(\omega)=$ $\left(z_{1}(\omega), \ldots, z_{\ell}(\omega)\right) \gg 0$ for each $\omega \in \Omega$.

Let the atomless measure $\eta(H)=\left(\int_{H}(g(t, \cdot)-e(t, \cdot)) \mathrm{d} \mu(t), \mu(H)\right)$, restricted to $A$. Applying Lyapunov theorem to $\eta$, we obtain that for any $\varepsilon$, with $0<\varepsilon<1$, there exists a coalition $\bar{A} \subset A$, with $\mu(\bar{A})=\varepsilon \mu(A)$, that privately blocks $f$ via $g$. This proves the result for $\alpha \leq \mu(A)$.

Otherwise, by convexity of preferences, for every $\varepsilon$, with $0<\varepsilon<1, V_{t}(\varepsilon g(t, \cdot)+(1-$ $\varepsilon) f(t, \cdot))>V_{t}(f(t, \cdot))$ for every $t \in A$.

Applying again Lyapunov convexity theorem, there exists $B \subset I \backslash A$, such that $\mu(B)=$ $(1-\varepsilon) \mu(I \backslash A)$ and

$$
\int_{B}(g(t, \cdot)-e(t, \cdot)) \mathrm{d} \mu(t)=(1-\varepsilon) \int_{I \backslash A}(g(t, \cdot)-e(t, \cdot)) \mathrm{d} \mu(t) .
$$

Let $\delta=\left(\delta_{1}, \ldots, \delta_{\ell}\right) \in \mathbb{R}_{+}^{\ell}$ given by $\delta_{j}=\min \left\{z_{j}(\omega), \omega \in \Omega\right\}$. Since preferences are convex and monotone, the coalition $S=A \bigcup B$ privately blocks $f$ via the allocation $h$ given by

$$
h(t, \cdot)= \begin{cases}\varepsilon g(t, \cdot)+(1-\varepsilon) f(t, \cdot) & \text { if } t \in A \\ f(t, \cdot)+\frac{\varepsilon}{\mu(B)} \delta & \text { if } t \in B\end{cases}
$$

Since $\mu(S)=\mu(A)+(1-\varepsilon) \mu(I \backslash A)$, we have constructed an arbitrarily large coalition privately blocking $f$.

\section{Equivalence result}

In order to obtain our main result, we introduce some additional notation. Given an allocation $x \in L_{X}$ and a vector $a=\left(a_{1}, \ldots, a_{n}\right)$, with $0 \leq a_{i} \leq 1$, let $\mathcal{E}(a, x)$ be a differential 
information exchange economy which coincides with $\mathcal{E}$ except for the initial endowment of each agent $i$ that is given by the following convex combination of $e_{i}$ and $x_{i}$ :

$$
e_{i}\left(a_{i}, x_{i}\right)=a_{i} e_{i}+\left(1-a_{i}\right) x_{i}
$$

That is,

$$
\mathcal{E}(a, x) \equiv\left\{\left((\Omega, \mathcal{F}), X_{i}, \mathcal{F}_{i}, U_{i}, e_{i}\left(a_{i}, x_{i}\right)=a_{i} e_{i}+\left(1-a_{i}\right) x_{i}, q\right): i=1, \ldots, n\right\}
$$

The next theorem states that a feasible allocation $x$ is an equilibrium allocation for $\mathcal{E}$ if and only if it is not privately blocked by the grand coalition in any economy $\mathcal{E}(a, x)$.

Theorem 4.1. Let $x$ be a feasible allocation in a differential information exchange economy $\mathcal{E}$ satisfying assumptions (A.1)-(A.3). Then $x$ is a Radner equilibrium allocation in $\mathcal{E}$ if and only if $x$ is a privately non-dominated allocation for every economy $\mathcal{E}(a, x)$.

Proof. Let $(p, x)$ be a Radner equilibrium for the economy $\mathcal{E}$. Suppose that there exists $a=\left(a_{1}, \ldots, a_{n}\right)$, such that $x$ is dominated in the economy $\mathcal{E}(a, x)$. Then, there exists $y=$ $\left(y_{1}, \ldots, y_{n}\right)$ such that

(i) $\sum_{i=1}^{n} y_{i} \leq \sum_{i=1}^{n} e_{i}\left(a_{i}, x_{i}\right)$, and

(ii) $V_{i}\left(y_{i}\right)>V_{i}\left(x_{i}\right)$ for every agent $i \in\{1, \ldots, n\}$.

Since $x$ is an Radner equilibrium allocation in the economy $\mathcal{E}$, we have that $\sum_{\omega \in \Omega} p(\omega)$. $x_{i}(\omega) \leq \sum_{\omega \in \Omega} p(\omega) \cdot e_{i}(\omega)$ for every agent $i$, and from condition (ii), we deduce that $\sum_{\omega \in \Omega} p(\omega) \cdot y_{i}(\omega)>\sum_{\omega \in \Omega} p(\omega) \cdot e_{i}(\omega)$, for every agent $i=1, \ldots, n$. Multiplying these inequalities by $\left(1-a_{i}\right)$ and by $a_{i}$, respectively, we obtain that

$$
\begin{aligned}
& \sum_{\omega \in \Omega} p(\omega) \cdot\left(1-a_{i}\right) y_{i}(\omega)>\sum_{\omega \in \Omega} p(\omega) \cdot\left(1-a_{i}\right) x_{i}(\omega) \text { and } \\
& \sum_{\omega \in \Omega} p(\omega) \cdot a_{i} y_{i}(\omega)>\sum_{\omega \in \Omega} p(\omega) \cdot a_{i} e_{i}(\omega) .
\end{aligned}
$$

Thus, $\sum_{\omega \in \Omega} p(\omega) \cdot y_{i}(\omega)>\sum_{\omega \in \Omega}\left(p(\omega) \cdot a_{i} e_{i}(\omega)+p(\omega) \cdot\left(1-a_{i}\right) x_{i}(\omega)\right)$ for every agent $i$. Therefore, $\sum_{i=1}^{n} \sum_{\omega \in \Omega} p(\omega) \cdot y_{i}(\omega)>\sum_{i=1}^{n} \sum_{\omega \in \Omega} p(\omega) \cdot e_{i}\left(a_{i}, x_{i}\right)(\omega)$, which is a contradiction with (i), that is, a contradiction with the feasibility of $y$ in the economy $\mathcal{E}(a, x)$.

Let $x$ be a privately non-dominated allocation for every economy $\mathcal{E}(a, x)$. Let $f$ be the step function on the real interval $I=[0,1]$, defined by $f(t)=x_{i}$ if $t \in I_{i}=\left[\frac{i-1}{n}, \frac{i}{n}\right)$, if $i \neq n$, and $f(t)=x_{n}$ if $t \in I_{n}=\left[\frac{n-1}{n}, 1\right]$.

Assume that $x$ is not a Radner equilibrium allocation for the economy $\mathcal{E}$. Then, by Theorem 3.1, the step allocation $f$ given by $x$ is not a Radner equilibrium allocation for the associated continuum economy $\mathcal{E}_{\mathrm{c}}$ with $n$ different types of agents. Applying the equivalence between the private core and the set of Radner equilibrium allocations (see Einy et al., 2001), we have that $f$ does not belong to the private core of the associated continuum 
economy. Furthermore, by Proposition 3.1, there exists a coalition $S \subset I=[0,1]$, with $\mu(S)>1-\frac{1}{n}$, and there exists $g: S \times \Omega \rightarrow \mathbb{R}_{+}^{\ell}$, such that $g(t, \cdot)$ is $\mathcal{F}_{t}$-measurable for every $t \in S ; \int_{S} g(t, \cdot) \mathrm{d} \mu(t) \leq \int_{S} e(t, \cdot) \mathrm{d} \mu(t)$ and $V_{t}(g(t, \cdot))>V_{t}(f(t, \cdot))$ for all $t \in S$. Let $S_{i}=S \bigcap I_{i}$ and $a_{i}=n \mu\left(S_{i}\right)$. Notice that, since $\mu(S)>1-\frac{1}{n}$, we obtain that $a_{i}>0$ for every $i$.

In the finite economy $\mathcal{E}$, let us consider the allocation $\left(g_{1}, \ldots, g_{n}\right)$, where $g_{i}=$ $\frac{1}{\mu\left(S_{i}\right)} \int_{S_{i}} g(t, \cdot) \mathrm{d} \mu(t)$. Since $g(t, \cdot)$ is $\mathcal{F}_{t}$-measurable for every $t \in S$, the function $g_{i}$ is $\mathcal{F}_{i^{-}}$ measurable for every $i=1, \ldots, n$.

Let $z_{i}=a_{i} g_{i}+\left(1-a_{i}\right) x_{i}$. By construction, $\sum_{i=1}^{n} z_{i} \leq \sum_{i=1}^{n} a_{i} e_{i}+\left(1-a_{i}\right) x_{i}$. By convexity of preferences, $V_{i}\left(z_{i}\right)>V_{i}\left(x_{i}\right)$, for every agent $i \in\{1, \ldots, n\}$.

Therefore, the grand coalition privately blocks $x$ via $z$ in the economy $\mathcal{E}(a, x)$, which is a contradiction.

\section{Concluding remarks}

Remark 1. It is important to notice that, since the deterministic A-D-M model is a special case of the differential information economy model, the equivalence result (Theorem 4.1) stated in the previous section allows us to obtain a new characterization of the Walrasian equilibria.

Let $\Gamma=\left\{\mathbb{R}_{+}^{\ell},\left(U_{i}, e_{i}\right): i=1, \ldots, n\right\}$ be an exchange economy, where $\mathbb{R}_{+}^{\ell}$ is the consumption set of every agent $i, U_{i}: \mathbb{R}_{+}^{\ell} \rightarrow \mathbb{R}$ the utility function of agent $i$ and $e_{i} \in \mathbb{R}_{+}^{\ell}$ is the initial endowment of agent $i$.

Given an allocation $x=\left(x_{1}, \ldots, x_{n}\right) \in \mathbb{R}_{+}^{\ell n}$ and a vector $a=\left(a_{1}, \ldots, a_{n}\right)$ with $0 \leq$ $a_{i} \leq 1$, let $\Gamma(a, x)$ be an exchange economy which is the same with $\Gamma$ except for the initial endowment of each agent $i$ which is given by the following convex combination of $e_{i}$ and $x_{i}: e_{i}\left(a_{i}, x_{i}\right)=a_{i} e_{i}+\left(1-a_{i}\right) x_{i}$. Thus, $\Gamma(a, x)=\left\{\mathbb{R}_{+}^{\ell},\left(U_{i}, e_{i}\left(a_{i}, x_{i}\right)\right): i=1, \ldots, n\right\}$.

In the scenario of a pure exchange economy, and following Definition 2.2, an allocation $x$ (feasible or not) is said to be dominated in the economy if there exists a feasible allocation such that every agent becomes better off. Observe that to be feasible and to be dominated are independent conditions. A Pareto optimal allocation is a feasible and non-dominated allocation.

As a consequence of Theorem 4.1, we obtain as corollary the following characterization of Walrasian equilibria:

Corollary. Suppose that in the economy $\Gamma$ initial endowments are strictly positive and utility functions are continuous, strictly monotone and concave. Let $x$ be a feasible allocation in $\Gamma$. Then, $x$ is a Walrasian equilibrium allocation in $\Gamma$ if and only if $x$ is non-dominated in every economy $\Gamma(a, x)$.

Remark 2. The characterization of Radner equilibria (and, in particular, of Walrasian equilibria) provided in this paper is independent of prices. The classical equivalence results, Debreu and Scarf (1963) and Aubin (1979), characterize Walrasian allocations by means of the core of an economy and, therefore, are also independent of prices. Precisely, the result by 
Debreu and Scarf (1963) characterizes the Walrasian allocations as those allocations which belong to the core of every $n$-fold replica economy, and the result of Aubin (1979) shows that the set of Walrasian allocations in a fixed finite economy coincides with the fuzzy core. However, our equivalence result differs from both characterizations of Walrasian equilibria. Debreu-Scarf consider the blocking power of coalitions in any replica economy and Aubin considers that every agent can participate not only with her total endowment but also with a fraction. Therefore, Debreu and Scarf (1963) as well as Aubin (1979) enlarge the set of blocking coalitions in order to get their characterization results whereas our equivalence result requires only the blocking power of the grand coalition (in a precise set of economies) in order to characterize equilibria.

Remark 3. It should be noted that we characterize the Radner equilibria allocations (and, in particular, Walrasian allocations) as those non-dominated allocations in the economies given by infinitesimal perturbations in a precise direction of the original endowments. In fact, the parameters $a_{i}$ in the statement of Theorem 4.1 can be chosen arbitrarily close to 1 for every agent $i$. Indeed, note that given $\delta$, with $0<\delta<1$, it is enough to consider the privately blocking coalition $S$ such that $\mu(S)>1-\frac{\delta}{n}$ in order to guarantee $a_{i}=n \mu\left(S_{i}\right)>1-\delta$ for every $i$.

Remark 4. Notice that the first welfare theorem is an immediate consequence of Theorem 4.1. In fact, if $x$ is a Radner or Walrasian equilibrium allocation, then $x$ is a Pareto optimal allocation not only in the economy $\mathcal{E}$ but also in any economy $\mathcal{E}(a, x)$ where $x$ is feasible.

On the other hand, observe that if $x$ is a Pareto optimal allocation in $\mathcal{E}$, then $x$ is also a Pareto optimal allocation in the economy in which the initial endowment allocation is $x$, that is, in the economy $\mathcal{E}(0, x)$. Thus, by taking $x_{i}=e_{i}$, for all $i$, all the economies $\mathcal{E}(a, x)$ are equal to $\mathcal{E}(0, x)$ and $x$ is not blocked by the grand coalition. Then, if $x \gg 0$ (i.e., if $x$ is strictly positive), we can apply Theorem 4.1 to the economy $\mathcal{E}(0, x)$ and we obtain, exactly, the second welfare theorem.

Therefore, both welfare theorems are particular cases of Theorem 4.1.

Remark 5. As it was remarked in Section 2, we have allowed for free disposal since a Radner equilibrium may not exist with positive prices. However, by allowing for negative prices, one can dispense with the free disposal assumption. Notice that the private core equivalence theorem of Einy et al. (2001) does not depend on whether or not the free disposal assumption holds. Indeed, as it is the case in the Aumann $(1964,1966)$ deterministic model with a continuum of agents, the assumptions which guarantee the core-Walras equivalence may not ensure nonemptiness of the sets. The results obtained in this paper still hold without the free disposal assumption, but in order to guarantee the existence of Radner equilibrium, we must allow for negative prices (see Radner, 1968).

Remark 6. The reader may wonder (and indeed the referee asked the question) if the results of this paper hold in the presence of a continuum of states. Notice that with a continuum of states, the dimensionality of the commodity spaces becomes infinite, and thus, the Laypunov theorem which is used for the proof of Vind's theorem fails. Thus, our method of proof cannot be readily extended to cover the continuum of states. 


\section{Acknowledgements}

The authors are grateful to an anonymous referee for his/her careful reading and helpful comments and suggestions. C. Hervés and E. Moreno acknowledge support by Research Grants BEC2000-1388-C04-01 (Ministerio de Ciencia y Tecnología and FEDER) and SA091/02 (Junta de Castilla y León).

\section{Appendix A}

Proof of Lemma 3.2. Let $f$ be an allocation which does not belong to the private core of $\mathcal{E}_{\mathrm{c}}$. Then, there exist a coalition $A$ and a function $h: A \times \Omega \rightarrow \mathbb{R}_{+}^{\ell}$, such that $h(t, \cdot)$ is $\mathcal{F}_{t}$-measurable for every $t \in A, \int_{A} h(t, \omega) \mathrm{d} \mu(t) \leq \int_{A} e(t, \omega) \mathrm{d} \mu(t)$ for every $\omega \in \Omega$ and $V_{t}(h(t, \cdot))>V_{t}(f(t, \cdot))$ for every agent $t \in A$.

Let us show that we can take $h$ such that $\int_{A} h(t, \omega) \mathrm{d} \mu(t) \gg 0$ for every $\omega \in \Omega$. For each commodity $j$ and each state $\omega \in \Omega$, let us define

$$
H(j, \omega)=\left\{t \in A \text { such that } h_{j}(t, \omega)=0\right\} .
$$

Assume that there exists $\left(j_{0}, \omega_{0}\right)$ such that $\mu\left(H\left(j_{0}, \omega_{0}\right)\right)=\mu(A)$. This implies that there is a type of agents $i_{0}$ such that $h_{j_{0}}\left(t, \omega_{0}\right)=0$ for every $t \in A_{i_{0}}=A \bigcap I_{i_{0}}$. Since $h(t, \cdot)$ is $\mathcal{F}_{t}$-measurable for every $t \in A, h_{j_{o}}(t, \omega)=0$, for every $t \in A_{i_{0}}$ and for every $\omega \in E_{i_{0}}\left(\omega_{0}\right)$, where $E_{i_{0}}\left(\omega_{0}\right)$ is the subset of the partition $\mathcal{F}_{i_{0}}$ to which $\omega_{0}$ belongs.

Given $\omega \in E_{i_{0}}\left(\omega_{0}\right)$, either (i) $\int_{A \backslash A_{i_{0}}} h_{j_{0}}(t, \omega) \mathrm{d} \mu(t)=\int_{A} e_{j_{0}}(t, \omega) \mathrm{d} \mu(t)$ holds, or (ii) $\int_{A} e_{j_{0}}(t, \omega) \mathrm{d} \mu(t)-\int_{A \backslash A_{i_{0}}} h_{j_{0}}(t, \omega) \mathrm{d} \mu(t)=\varepsilon(\omega)>0$ holds.

Let $\Upsilon=\left\{\omega \in E_{i_{0}}\left(\omega_{0}\right)\right.$ such that (i) holds $\}$. If $\omega \in \Upsilon$, there exists a type of agents $i(\omega)$ such that $\int_{A} \bigcap_{i(\omega)} h_{j_{0}}(t, \omega) \mathrm{d} \mu(t)>0$. Then, applying Lusin's theorem, we have that for each $\omega \in \Upsilon$ there exists a compact set $B(\omega) \subset A_{i(\omega)}=A \bigcap I_{i(\omega)}$, with $\mu(B(\omega))>$ $\mu\left(A_{i(\omega)}\right)-\varepsilon$, such that $f(\cdot, \omega)$ and $h(\cdot, \omega)$ are continuous functions on $B(\omega)$. Hence, by continuity of preferences, there exists $b>0$ such that $U_{i(\omega)}(h(\cdot, \omega))-b>U_{i(\omega)}(f(\cdot, \omega))+b$ for every $t \in B(\omega)$. Then, there exists $\delta(\omega)>0$, such that $V_{t}(\hat{h}(t, \cdot))>V_{t}(f(t, \cdot))$ for every $t \in B=\bigcup_{\omega \in \Upsilon} B(\omega)$, where

$$
\hat{h}_{j}(t, \omega)= \begin{cases}h_{j_{0}}(t, \omega)-\delta(\omega) & \text { if } j=j_{0}, \omega \in \Upsilon \text { and } t \in B(\omega) \\ h_{j}(t, \omega) & \text { otherwise. }\end{cases}
$$

Let $\beta=\min _{\omega \in E_{i_{0}}\left(\omega_{0}\right)}\left\{(\varepsilon(\omega))_{\omega \notin \Upsilon},(\delta(\omega) \mu(B(\omega)))_{\omega \in \Upsilon}\right\}$. Note that $\beta$ is a strictly positive real number. Let us define the allocation $\hat{g}: A \times \Omega \rightarrow \mathbb{R}_{+}^{\ell}$ as follows:

$$
\hat{g}_{j}(t, \omega)= \begin{cases}\hat{h}_{j}(t, \omega) & \text { if } \omega \in \Upsilon \text { and } t \in B \\ \frac{\beta}{\mu\left(A_{i_{0}}\right)} & \text { if } j=j_{0}, \omega \in E_{i_{0}}\left(\omega_{0}\right) \text { and } t \in A_{i_{0}} \\ h_{j}(t, \omega) & \text { otherwise. }\end{cases}
$$


By construction, $A$ privately blocks $f$ via $\hat{g}$ and $\int_{A} \hat{g}_{j_{0}}(t, \omega) \mathrm{d} \mu(t)>0$ for every $\omega \in$ $E_{i_{0}}\left(\omega_{0}\right)$.

Applying the argument above as many times as necessary, we can take $\bar{g}$ such that $A$ blocks $f$ via $\bar{g}$ and $\int_{A} \bar{g}(t, \omega) \mathrm{d} \mu(t) \gg 0$, for every $\omega$. If the inequality $\int_{A} \bar{g}(t, \cdot) \mathrm{d} \mu(t) \ll \int_{A} e(t, \cdot) \mathrm{d} \mu(t)$ does not hold, there exist a commodity $j_{1}$ and a state $\omega_{1}$ such that $\int_{A} \bar{g}_{j_{1}}\left(t, \omega_{1}\right) d \mu(t)=\int_{A} e_{j_{1}}\left(t, \omega_{1}\right) \mathrm{d} \mu(t)$. Then, for some type $i$ of agents $\bar{g}_{j_{1}}\left(t, \omega_{1}\right)>0$ for every $t \in B_{i} \subset A_{i}$, with $\mu\left(B_{i}\right)>0$. By continuity of preferences, we can take $\varepsilon: B_{i} \rightarrow \mathbb{R}_{+}$, with $\int_{B_{i}} \varepsilon(t) \mathrm{d} \mu(t)>0$, such that the coalition $A$ privately blocks $f$ via the allocation $g$ given by

$$
g_{j}(t, \omega)= \begin{cases}\bar{g}_{j_{1}}\left(t, \omega_{1}\right)-\varepsilon(t) & \text { if } t \in B_{i}, j=j_{1} \text { and } \omega \in E_{i}\left(\omega_{1}\right) \\ \bar{g}_{j}(t, \omega) & \text { otherwise. }\end{cases}
$$

Now, consider the set $\left\{(j, \omega) \in(\{1, \ldots, \ell\} \times \Omega) \backslash\left(\left\{j_{1}\right\} \times E_{i}\left(\omega_{1}\right)\right)\right\}$ and apply the previous argument. In the same way, we can construct an allocation $g$ such that $A$ privately blocks $f$ via $g$ and $\int_{A} g(t, \omega) \mathrm{d} \mu(t) \ll \int_{A} e(t, \omega) \mathrm{d} \mu(t)$ for every state $\omega \in \Omega$.

\section{References}

Aubin, J.P., 1979. Mathematical Methods of Game Economic Theory. North-Holland, Amsterdam, New York, Oxford.

Aumann, R.J., 1964. Markets with a continuum of traders. Econometrica 32, 39-50.

Aumann, R.J., 1966. Existence of competitive equilibria in markets with a continuum of traders. Econometrica $34,1-17$.

Debreu, G., 1959. Theory of Value. Yale University Press, New Haven.

Debreu, G., Scarf, H., 1963. A Limit theorem on the core of an economy. International Economic Review 4, $235-246$.

Einy, E., Moreno, D., Shitovitz, B., 2001. Competitive and core allocations in large economies with differentiated information. Economic Theory 18, 321-332.

García-Cutrín, F.J., Hervés-Beloso, C., 1993. A discrete approach to continuum economies. Economic Theory 3, 577-583.

Glycopantis, D., Muir, A., Yannelis, N.C., 2003. On extensive form implementation of contracts in differential information economies. Economic Theory 21, 495-526.

Grodal, B., 1972. A second remark on the core of an atomless economy. Econometrica 40, 581-583.

Kreps, D., 1977. A note on fulfilled expectations equilibria. Journal of Economic Theory 14, 32-43.

Radner, R., 1968. Competitive equilibrium under uncertainty. Econometrica 36, 31-58.

Radner, R., 1982. Equilibrium under uncertainty. In: Arrow, K.J., Intriligator, M.D. (Eds.), Handbook of Mathematical Economics, vol. II. Amsterdam, North Holland.

Radner, R., 1979. Rational expectations equilibrium: generic existence and the information revealed by prices. Econometrica 47, 655-678.

Schmeidler, D., 1972. A remark on the core of an atomless economy. Econometrica 40, 579-580.

Vind, K., 1972. A third remark on the core of an atomless economy. Econometrica 40, 585-586.

Yannelis, N.C., 1991. The core of an economy with differential information. Economic Theory 1, 183-198. 